\title{
COMPET TALKS: O CICLO DE PALESTRAS REALIZADAS PELO COMPET
}

Marcus V Durães Zuba - marcusduraes2010@gmail.com

Thereza R de Faria Morais - therezarfaria@gmail.com

André R da Cruz - dacruz@cefetmg.br

Joana A P Forte - joanaforte@cefetmg.br

Sandro R Dias - sandrord@cefetmg.br

Centro Federal de Educação Tecnológica de Minas Gerais/Campus II

Avenida Amazonas, 7675, Nova Gameleira

CEP 30510-000 - Belo Horizonte - Minas Gerais

Resumo: Sabendo-se que uma das responsabilidades do trabalho PET é aumentar o engajamento dos estudantes com o curso de graduação, o Grupo PET de Engenharia de Computação do CEFET-MG, COMPET, organiza e oferece para a comunidade cefetiana o COMPET Talks. Esta atividade visa oferecer, através de palestras curtas, um conteúdo atual sobre ciência, tecnologia e mercado de trabalho, a fim de motivar os acadêmicos nos estudos e consequentemente diminuir a evasão do curso. Para isso, são convidados profissionais e acadêmicos de renome com trabalhos reconhecidos em suas áreas de atuação. Até o momento foram ofertadas seis palestras, com temas diversos dentro da computação, que tiveram uma ótima avaliação quanto ao conteúdo e refletiram um grande interesse dos participantes presentes. Diante disso, o objetivo deste artigo é avaliar a proposta do evento e analisar quantitativamente e qualitativamente o seu impacto na comunidade acadêmica do CEFET-MG, por meio da coleta de avaliações do público participante, chegando-se à conclusão que houve um impacto positivo no engajamento dos estudantes do curso de Engenharia de Computação, reduzindo assim a potencial evasão do curso pelos alunos.

Palavras-chave: Palestras. Palestras curtas. Educação. Computação. Engajamento acadêmico.

\section{INTRODUÇÃO}

A evasão dos cursos de engenharia sempre foi uma problemática relevante no contexto acadêmico do Brasil. Conforme estudos realizados na área, um dos principais motivos que influenciam um estudante de ensino superior a desistir da graduação e sair do seu curso é a falta de participação em atividades externas à sala de aula (BARDAGI, 2007). Diante desse cenário, é de suma importância a ação dos grupos PET, que, dentre várias outras responsabilidades, devem buscar uma melhora no engajamento dos estudantes para com o curso. Analisando pesquisas efetuadas sobre o tema, é possível concluir que o engajamento acadêmico é considerado a variável mais influente nos resultados de aprendizagem e aderência aos estudos (ZEPKE, 2011).

Diante disso, é essencial que sejam realizadas ações fora da sala de aula no ambiente da universidade, por exemplo palestras, que visem a uma maior participação das pessoas envolvidas no contexto acadêmico e que, consequentemente, afetem positivamente nos índices de engajamento dos estudantes (VITÓRIA et al., 2018). Com esse objetivo em mente, 


\section{COBENGE (C) COBENGE 2020

o COMPET decidiu promover para a comunidade acadêmica do CEFET-MG o COMPET Talks. Este trabalho tem como objetivo examinar o planejamento do evento, bem como as ações que já foram executadas, além de avaliar os seus efeitos no contexto universitário do CEFET-MG.

Inspirado no modelo das palestras curtas com temas inovadores do TED Talks (ANDERSON, 2016), o COMPET Talks é uma série de palestras de até 30 minutos abertas para alunos e funcionários do CEFET-MG em que profissionais e pesquisadores da área de tecnologia apresentam novas pesquisas, descobertas e ferramentas e discutem esses temas com o público.

O COMPET Talks possui como principal atividade em cada evento uma apresentação de temas inovadores para os alunos, professores e técnicos administrativos de maneira dinâmica, reservando 20 minutos para a exposição do tema e 10 minutos para a resposta a quaisquer dúvidas que tenham surgido. A opção por este formato se deu por ser um espaço de tempo suficiente para evitar a distração dos participantes, como ocorre em palestras de longa duração, enquanto ainda o bastante para apresentar o tema de maneira concreta (ANDERSON, 2016).

A expectativa é que esses temas inovadores provoquem uma motivação nos alunos, tanto naqueles que cursam engenharia de computação quanto dos visitantes de outras áreas, implicando um maior interesse no estudo de assuntos fundamentais da área de computação e, consequentemente, aumentando a participação dos acadêmicos nas atividades que são desenvolvidas ao longo da graduação.

As atividades já realizadas do COMPET Talks ocorreram em 2019 nos dias 28/03, 29/04, 31/05, 22/10; e em 2020 nos dias 24/04 e 29/05 e tiveram como temas: "O Cluster do CEFETMG e como utilizar adequadamente seus recursos", "Aprendizado e planejamento on-line em sistemas multi-agente", "Síntese de programas", "React - O que é e por que está dominando o mercado", "Marketing pessoal e o que as empresas de TI procuram" e "FrontEnd - O que é e por onde começar".

\section{MATERIAIS E MÉTODOS}

Para a execução dos eventos, são contactados palestrantes de diferentes áreas, levantado o horário em que teria-se uma adesão maior de espectadores e então organizado a estrutura para o palestrante. É divulgado um formulário de inscrição duas semanas anteriores às palestras, para que os interessados no evento possam preencher com os seus dados de contato (nome, telefone e email) e as suas informações acadêmicas, tais como o curso e o período que estão cursando no momento da inscrição.

A primeira palestra, ministrada por Felipe Duarte, servidor e aluno de mestrado do Programa de Pós-Graduação em Modelagem Matemática Computacional pelo CEFET-MG, ocorreu no dia 28/03/2019, com o tema "O Cluster do CEFET e como utilizar adequadamente seus recursos". A palestra abordou sobre a forma de organização de computadores cluster e seus conceitos. Além disso, também foi abordado, de maneira mais específica, o cluster alocado no Laboratório de Computação de Alto Desempenho do CEFET-MG.

A segunda palestra, "Aprendizado e planejamento on-line em sistemas multi-agente", ocorrida no dia 29/04/2019 e apresentada por Leandro Marcolino, tratou do aprendizado e planejamento on-line em problemas complexos envolvendo sistemas com vários agentes inteligentes. Foram abordadas especialmente as três linhas da pesquisa: "Agentes ou ações"; "Aprendendo e planejando contra enxames em tempo real" e "Times ad-hoc em neblina".

A terceira palestra ocorreu no dia 31/05/2019, com o assunto "Síntese de programas", por Gabriel Poesia, falando sobre a pesquisa que se propõe a gerar programas automaticamente dada uma especificação de mais alto nível, possivelmente incompleta e ambígua, como 
exemplos de entrada e saída ou até mesmo linguagem natural. Ele apresentou ideias fundamentais da área, algumas aplicações já existentes em softwares comerciais, bem como hipóteses sobre o futuro da programação.

A quarta palestra, ocorrida no dia 22/10/2019, com o tema "React - o que é e por que está dominando o mercado", foi ministrada pelo convidado Artur Bani, na qual ele expôs a renomada biblioteca de Javascript, que atualmente é muito utilizada no mercado para a criação de interfaces em páginas da Web. Na oportunidade ele fez uma apresentação teórica e também uma exposição prática da utilização da biblioteca, garantindo um melhor entendimento para o público presente.

A quinta palestra foi realizada no dia 24/04/2020, em uma parceria concretizada com a empresa de tecnologia da informação DTI Digital, cujo tema foi "Marketing Digital e o que as empresas de TI procuram". Essa palestra foi realizada de forma totalmente on-line, em virtude da pandemia do novo coronavírus, utilizando-se a plataforma Microsoft Teams. Na ocasião, as palestrantes Gabriela Vieira e Larissa Pacífico, ambas colaboradoras da DTI, explicaram o conceito básico de marketing digital, além de expor habilidades essenciais para profissionais que desejam se candidatar a processos seletivos em empresas de TI.

Por fim, a sexta palestra "FrontEnd: O que é e por onde começar" ocorreu no dia 29/05/2020. Assim como a anterior, esta também foi realizada de maneira totalmente virtual, por meio das plataformas Conferência Web e YouTube, haja vista o isolamento social vigente na época de execução do evento. O palestrante foi o estudante de Engenharia de Computação do CEFET-MG Pedro Lages, que realizou uma introdução ao contexto FrontEnd do desenvolvimento web, expondo as principais tecnologias usadas e fornecendo algumas dicas para quem está começando o aprendizado dessas ferramentas.

As palestras também foram gravadas e exibidas ao vivo no canal do COMPET no YouTube, com exceção da quinta palestra - pelo fato de ter sido uma parceria com um evento restrito de uma empresa privada da área de computação - e podem ser acessadas pelos links https://youtu.be/U3TqvZsuwbA, https://youtu.be/DCQKmUSefy0, https://youtu.be/uaaT31h 3G4A, https://youtu.be/W0xu4qx1hiw e https://youtu.be/Us2Ydu9F6lk. O público de tais atividades contou com a participação de técnicos administrativos, professores e alunos de curso técnico, graduação e pós-graduação, além de algumas pessoas externas na última palestra realizada, haja vista que, por ser on-line, não haveria a restrição de acesso para apenas alunos do CEFET-MG.

A respeito do processo de avaliação da atividade, a plataforma Google Forms foi utilizada para a criação e aplicação de formulários online. Esses formulários são compostos por três perguntas de múltipla escolha, com respostas que variam em 5 índices, sendo o mais baixo para muito ruim e o mais alto para muito bom. Essas perguntas são: "Qual o seu nível de satisfação com o evento?", "Qual a sua satisfação com os assuntos abordados?" e "Qual a sua nota para a organização do evento?". Além disso, o formulário contém dois campos de texto, que podem ser utilizados para críticas ou comentários e também para sugestão de temas futuros para o COMPET Talks.

A divulgação desses formulários é efetuada após o término das palestras, sendo o link de acesso ao formulário de avaliação enviado ao email dos participantes, utilizando como base o que foi informado por cada participante no momento da inscrição. Com isso, é possível realizar uma análise quantitativa das avaliações, a partir dos gráficos que podem ser gerados com as porcentagens de cada resposta de múltipla escolha; além de uma análise qualitativa das críticas e comentários fornecidos nos campos de texto. 


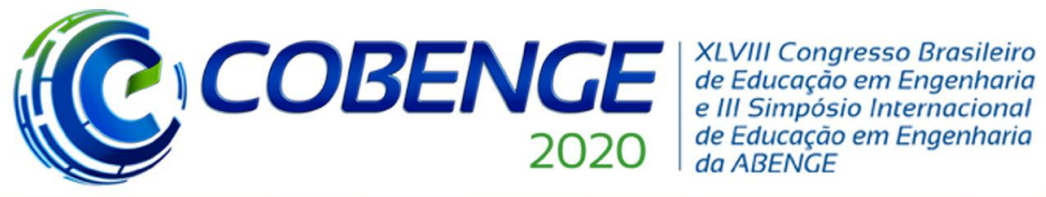

"Os desafios para formar hoje o engenheiro do amanhã"

\section{RESULTADOS E DISCUSSÃO}

Iniciando o processo de análise quantitativa dos resultados, é necessário observar as avaliações das primeiras palestras realizadas em 2019. Em primeiro lugar, tem-se um gráfico que compila as respostas da pergunta do formulário cujo objetivo é medir a satisfação do público com o conteúdo das palestras em si, exposto na figura 1. A partir da observação dos dados presentes no gráfico, com $100 \%$ das respostas sendo de índice máximo, conclui-se que o conteúdo do evento teve uma ótima recepção do público, fato esse que é fundamental para que haja um interesse contínuo das pessoas que comparecem às palestras realizadas, haja vista que a presença de bons temas incentivam os espectadores a continuarem acompanhando os próximos eventos que serão realizados.

Figura 1: Avaliação do conteúdo das primeiras palestras (0 para "Muito Ruim” até 5 para "Ótimo").

\section{De 0 a 5 dê uma nota à palestra que assistiu}

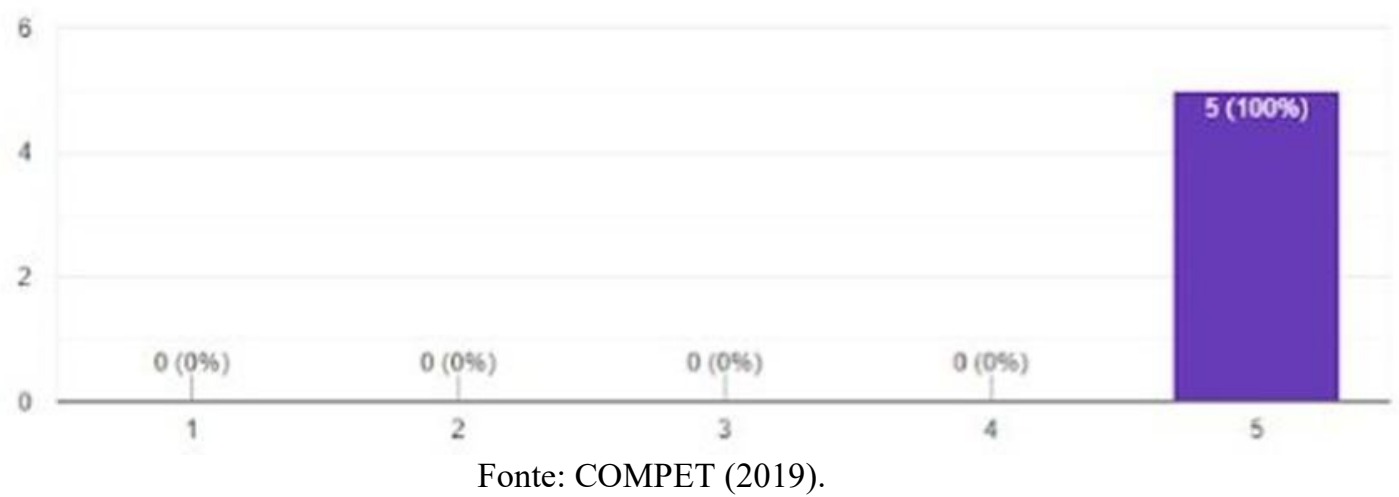

O gráfico da figura 2 compila as respostas a respeito da organização do evento. Analisando-o, é possível perceber que a maioria das respostas $(60 \%)$ foram de índice mediano, implicando que o grupo precisava melhorar quanto à organização e preparo do evento com mais antecedência e que a divulgação dentro do CEFET-MG precisava ser melhorada. $\mathrm{O}$ COMPET se mobilizou sobre essas avaliações modificando o horário para um mais adequado, obtido por meio de uma pesquisa realizada antes de cada evento com os estudantes do curso de Engenharia de Computação. Foi notado um resultado positivo após essa mudança, com uma adesão maior de ouvintes nas palestras realizadas posteriormente. 


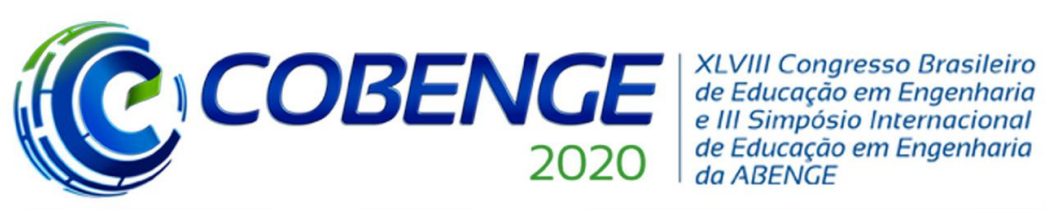

"Os desafios para formar hoje o engenheiro do amanhã"

Figura 2: Avaliação da organização das primeiras palestras (0 para "Muito Ruim" até 5 para "Ótimo").

De 0 a 5 dê uma nota à organização do evento

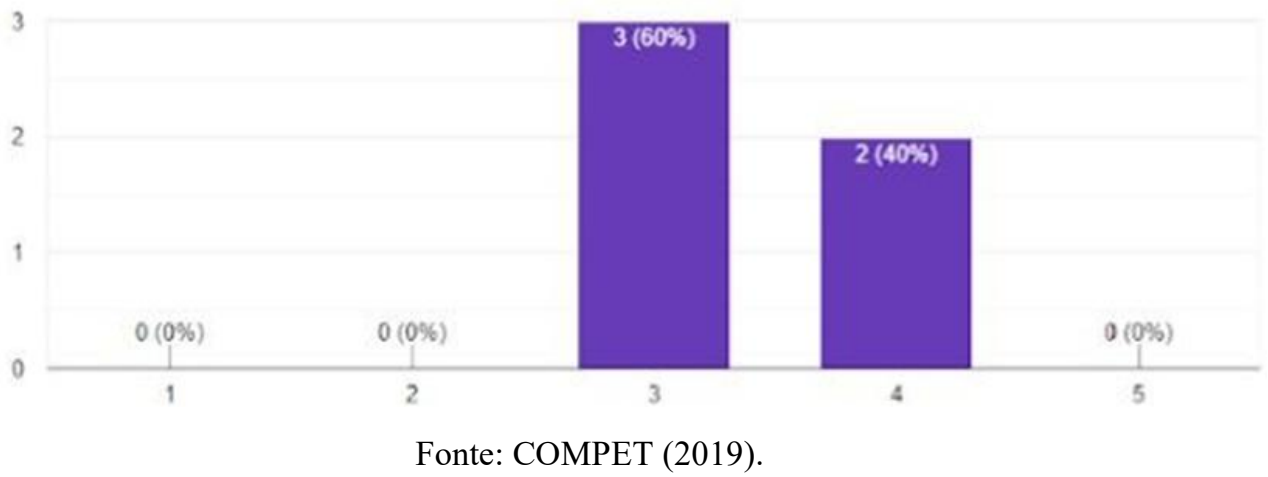

Analisando as avaliações e comentários das duas últimas palestras realizadas, primeiro considera-se o gráfico presente na figura 3, que compila as respostas da pergunta cujo objetivo é medir o nível de satisfação geral do público com o evento. Com base nos dados presentes no gráfico, dos quais $71,1 \%$ são do índice máximo de resposta, é possível concluir que as palestras foram realizadas com um alto nível de qualidade, sendo bem recebidas pelo público. Além disso, percebe-se um aumento do número de participantes do evento - foi constatado uma presença de 40 pessoas, diante de um público esperado de 48 inscritos - e um aumento, também, no número de avaliações recebidas no formulário, refletindo um grande engajamento dos espectadores com o evento e, consequentemente, com o ambiente acadêmico como um todo.

Figura 3: Avaliação geral das últimas palestras (0 para "Muito Ruim” até 5 para “Ótimo"). Qual o seu nivel de satisfação com o evento?

38 respostas

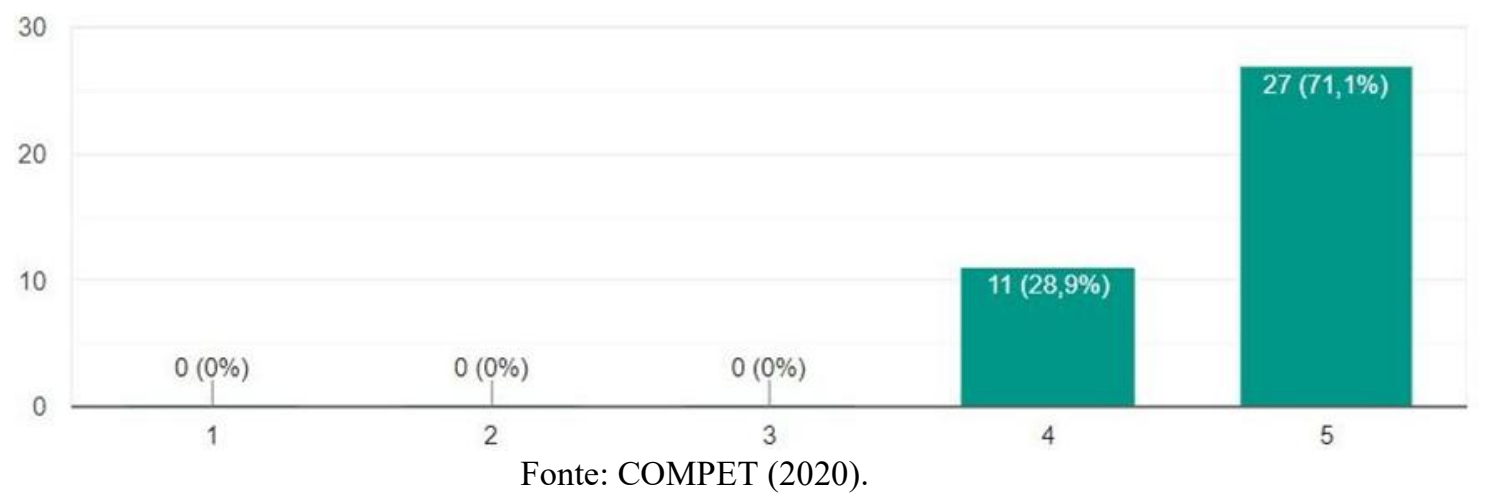

Além disso, é preciso analisar a satisfação do público participante das palestras a respeito dos assuntos abordados. Conforme o gráfico presente na figura 4, é possível notar que as temáticas abordadas foram bem recebidas pelo público, já que $73,7 \%$ das respostas obtiveram o índice máximo de satisfação. Em comparação com as avaliações recolhidas nas primeiras palestras, apesar da queda em porcentagem das respostas de nível máximo (que eram de $100 \%$ ), houve um aumento significativo na quantidade de avaliações coletadas, representando assim um grande incremento na quantidade de pessoas que ficaram muito satisfeitas com os 


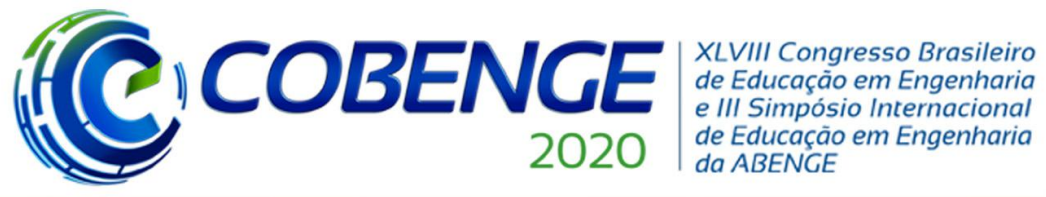

"Os desafios para formar hoje o engenheiro do amanhã"

assuntos abordados. Logo, é perceptível a grande qualidade dos temas abordados no COMPET Talks.

Figura 4: Avaliação do conteúdo das últimas palestras (0 para "Muito Ruim" até 5 para “Ótimo"). Qual a sua satisfação com os assuntos abordados?

38 respostas

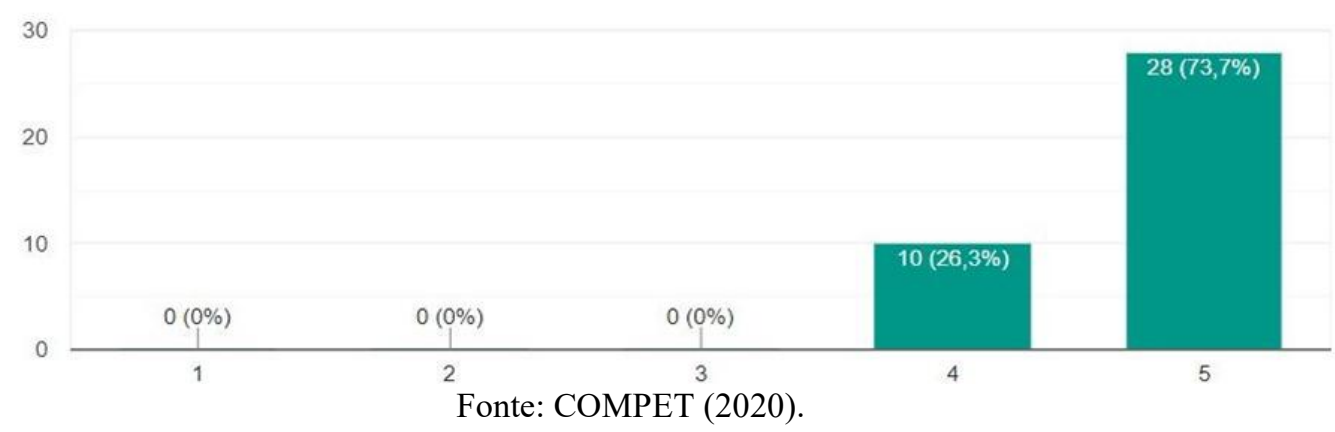

Finalizando a análise quantitativa das avaliações recolhidas, é necessário considerar como foi avaliado pelo público o aspecto da organização do evento. Diante do gráfico exposto na figura 5, que compila as respostas da pergunta do formulário que aborda esse aspecto, é possível perceber que a maioria dos participantes que responderam ao formulário de avaliação ficaram muito satisfeitos com a organização das palestras. Apesar de ainda possuir algumas poucas respostas que refletem baixo nível de satisfação, é notória a evolução da qualidade da organização realizada pelo COMPET, haja vista que a maioria das respostas passou de "3 (Neutro)" - resultado obtido nas avaliações das primeiras palestras - para "5 (Ótimo)", além de uma quantidade considerável de respostas "4 (Muito bom)". Esse fato é importante para que seja mantido um interesse constante do público, à medida que uma boa organização promove um maior conforto para os participantes, incentivando, assim, a continuidade das participações nas palestras realizadas.

Figura 5: Avaliação da organização das últimas palestras (0 para "Muito Ruim" até 5 para “Ótimo”). Qual sua nota para organização do evento?

38 respostas

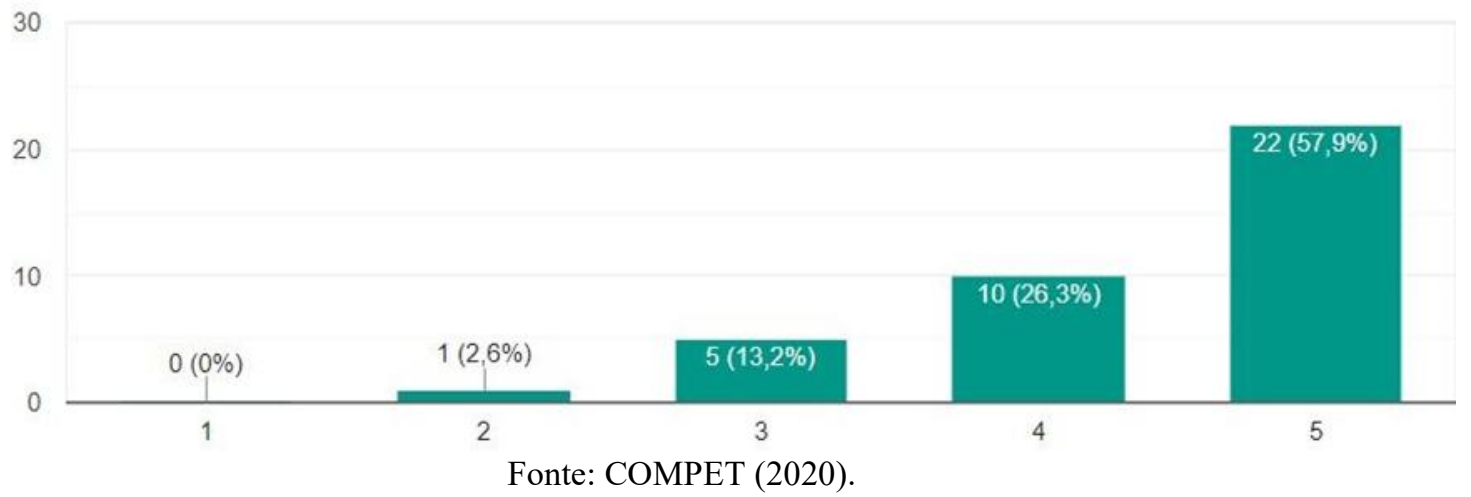

No que tange à análise qualitativa das atividades realizadas, que pode ser realizada a partir dos campos de texto presentes no formulário de avaliação, é possível perceber um intenso envolvimento do público com os eventos. O primeiro indício para isso é a grande quantidade de respostas fornecidas nesses campos de texto, que nas duas últimas palestras realizadas acumularam 22 críticas, comentários ou sugestões de temas futuros. 
A título de exemplo das críticas e comentários, cita-se: "seria legal se houvesse uma versão texto da apresentação", "A palestra foi ótima! Clima descontraído e com muita informação relevante!", "Acho que o link para o evento poderia ser enviado com maior antecedência.", "Somente elogiar a aula mesmo, parabéns aí.", dentre outros. A respeito das sugestões para temas futuros, foram fornecidas respostas como: "Desenvolvimento Mobile", "Segurança da informação, Redes e inteligência artificial", "Acho que seria bastante interessante algo focado especificamente em soft skills!", "Introdução a banco de dados" e outras temáticas.

Esses textos ratificam o resultado obtido nos gráficos e refletem uma preocupação dos participantes em contribuir para a constante melhoria da organização e desenvolvimento das palestras realizadas, além de colaborar no processo de seleção para temas futuros. Esse aspecto evidencia um interesse em continuar acompanhando os eventos, fato que certamente pode ser interpretado como impactante para um aumento do engajamento acadêmico das pessoas que participam das palestras.

Em suma, é possível concluir que o principal objetivo do COMPET Talks, este sendo melhorar o engajamento dos acadêmicos no curso de graduação, foi atingido. O público das palestras aumentou gradativamente à medida que os eventos ocorreram, bem como houve uma ampliação da participação das pessoas presentes nas avaliações e nas sugestões de novos temas. Isso reflete um nítido interesse em aprender cada vez mais, aspecto esse que, conforme a fundamentação teórica apresentada neste artigo, impacta positivamente na redução da evasão de estudantes.

É válido ressaltar também que a execução desse projeto contribuiu de forma relevante para um amadurecimento acadêmico do COMPET, à medida que foi possível adquirir conhecimentos e experiências importantes a respeito da organização de eventos educacionais. As experiências vivenciadas pelo grupo nas transmissões ao vivo dos eventos se mostraram essenciais para um grande aprendizado referente aos ambientes virtuais e o seu uso para a execução e propagação dos conteúdos desenvolvidos.

Por fim, devido ao grande sucesso do ciclo de palestras oferecido no COMPET Talks, planeja-se continuar com a execução desse trabalho, ao passo que já se tem estabelecido um cronograma que abrange até o final do ano de 2020, com eventos mensais, que serão executados ao longo de todo o ano letivo, totalizando oito palestras neste ano.

\section{CONSIDERAÇÕES FINAIS}

Considerando que eventos universitários externos à sala de aula intensificam o engajamento acadêmico, aspecto essencial no combate à evasão dos cursos de graduação, o COMPET ofereceu o ciclo de palestras denominado COMPET Talks.

As palestras foram realizadas com sucesso e muito bem recebidas pelo público participante, como se pode observar pelas avaliações realizadas, apesar das dificuldades presentes nos primeiros eventos oferecidos. Esses empecilhos encontrados pelo COMPET na organização e gerenciamento do evento mostraram-se importantes para que houvesse um grande ganho em termos de conhecimento para o grupo, fato que pode ser percebido na quantidade crescente de público do evento e também no aumento do interesse da comunidade acadêmica do CEFET-MG para as questões abordadas nas palestras e, consequentemente, para os estudos da graduação de uma forma geral.

Em suma, pode-se considerar que as atividades já realizadas do COMPET Talks atingiram os objetivos previamente traçados para a iniciativa, de melhorar o engajamento acadêmico dos estudantes. Devido a isso, o projeto possui um planejamento já estabelecido para todas as palestras que serão realizadas até o final do ano de 2020 , em forma de eventos 
"Os desafios para formar hoje o engenheiro do amanhã"

on-line diante do cenário da pandemia do COVID-19, a fim de continuar o impacto positivo na comunidade acadêmica do CEFET-MG.

\title{
Agradecimentos
}

Agradecemos ao CEFET-MG pelo apoio e fomento do grupo PET, através da Diretoria de Graduação, para a realização da atividade aqui descrita, bem como o auxílio financeiro para a participação e apresentação deste trabalho no COBENGE 2020. Aproveitamos para agradecer também aos colegas que colaboraram na realização da atividade.

\section{REFERÊNCIAS}

ANDERSON, Chris. TED Talks: The Official TED Guide to Public Speaking. Nova Iorque: Houghton Mifflin Harcourt, 2016.

BARDAGI, Marúcia Patta. Evasão e Comportamento Vocacional de Universitários: Estudos sobre o desenvolvimento de carreira na graduação. 2007. Tese (Doutorado) - Instituto de Psicologia, Universidade Federal do Rio Grande do Sul, 2007.

VITÓRIA, Maria Inês Côrte et al. Engajamento acadêmico: desafios para a permanência do estudante na educação superior. : desafios para a permanência do estudante na Educação Superior. Educação, [s.1.], v. 41, n. 2, p. 262-269, 17 set. 2018. EDIPUCRS. http://dx.doi.org/10.15448/1981-2582.2018.2.27960

ZEPKE, Nick. Student engagement research: thinking beyond the mainstream. : thinking beyond the mainstream. Higher Education Research \& Development, [s.1.], v. 34, n. 6, p. 1311-1323, 21 maio 2015. Informa UK Limited. http://dx.doi.org/10.1080/07294360.2015.1024635

\section{COMPET TALKS: THE LECTURES CYCLE PROMOTED BY COMPET}

\begin{abstract}
Knowing that one of the responsibilities of PET work is improve the engagement of undergraduate students, the CEFET-MG's Computer Engineering PET Group, COMPET, organize and offers to academic community the COMPET Talks. This activity aims offer, by means of short lectures, an updated content about science, technology and job market, to motivate the academics in their studies and consequently positively impact the evasion of the course. For that, reputed professionals and academics with known works in their actuation area are invited. So far, six lectures have been offered, with diverse themes in computer science, which had great evaluation about the content and reflected a great interest from the presents participants. Therefore, the objective of this article is to evaluate the event's proposal and to analyze quantitatively and qualitatively its impact on the academic community of CEFET-MG, through the collection of evaluations from the participating public, reaching the conclusion that there was a positive impact on the engagement of students in the Computer Engineering course, reducing the potential evasion of the course by students.
\end{abstract}

Keywords: lectures, short lectures, education, computer science, academic engagement. 